\title{
Representations for the Generalized Drazin Inverse of the Sum in a Banach Algebra and Its Application for Some Operator Matrices
}

\author{
Xiaoji Liu and Xiaolan Qin \\ Faculty of Science, Guangxi University for Nationalities, Nanning 530006, China \\ Correspondence should be addressed to Xiaoji Liu; xiaojiliu72@126.com \\ Received 26 September 2014; Accepted 8 January 2015 \\ Academic Editor: Predrag S. Stanimirovic
}

Copyright @ 2015 X. Liu and X. Qin. This is an open access article distributed under the Creative Commons Attribution License, which permits unrestricted use, distribution, and reproduction in any medium, provided the original work is properly cited.

We investigate additive properties of the generalized Drazin inverse in a Banach algebra $\mathscr{A}$. We find explicit expressions for the generalized Drazin inverse of the sum $a+b$, under new conditions on $a, b \in \mathscr{A}$. As an application we give some new representations for the generalized Drazin inverse of an operator matrix.

\section{Introduction}

Let $\mathscr{A}$ be a complex Banach algebra with unite 1 . We use $\sigma(a)$ to denote the spectrum of $a \in \mathscr{A}$. The sets of all nilpotent and quasinilpotent elements $(\sigma(a)=\{0\})$ of $\mathscr{A}$ will be denoted by $\mathscr{A}^{\text {nil }}$ and $\mathscr{A}^{\text {qnil }}$, respectively.

The generalized Drazin inverse of $a \in \mathscr{A}$ (introduced by Koliha in [1]) is the element $b \in \mathscr{A}$ which satisfies

$$
x a x=x, \quad a x=x a, \quad a-a^{2} x \in \mathscr{A}^{\text {qnil }} .
$$

If there exists the generalized Drazin inverse, then the generalized Drazin inverse of $a$ is unique and is denoted by $a^{d}$. The set of all generalized Drazin invertible elements of $\mathscr{A}$ is denoted by $\mathscr{A}^{d}$. For interesting properties of the generalized Drazin inverse see [2-6]. For a complete treatment of the generalized Drazin inverse, see [7, Chapter 2].

If $p=p^{2} \in \mathscr{A}$ is an idempotent, we denote $\bar{p}=\mathbb{1}-p$. We can represent element $a \in \mathscr{A}$ as

$$
a=\left[\begin{array}{ll}
a_{11} & a_{12} \\
a_{21} & a_{22}
\end{array}\right]_{p},
$$

where $a_{11}=p a p, a_{12}=p a \bar{p}, a_{21}=\bar{p} a p$, and $a_{22}=\bar{p} a \bar{p}$.
Let $a \in \mathscr{A}^{d}$ and $a^{\pi}=\mathbb{1}-a a^{d}$ be the spectral idempotent of $a$ corresponding to $\{0\}$. It is well known that $a \in \mathscr{A}$ can be represented in the following matrix form ([7, Chapter 2]):

$$
a=\left[\begin{array}{cc}
a_{1} & 0 \\
0 & a_{2}
\end{array}\right]_{p},
$$

relative to $p=a a^{d}$, where $a_{1}$ is invertible in the algebra $p \mathscr{A} p, a^{d}$ is its inverse in $p \mathscr{A} p$, and $a_{2}$ is quasinilpotent in the algebra $\bar{p} \mathscr{A} \bar{p}$. Thus, the generalized Drazin inverse of $a$ can be expressed as

$$
a^{d}=\left[\begin{array}{cc}
a_{1}^{d} & 0 \\
0 & 0
\end{array}\right]_{p}
$$

Obviously, if $a \in \mathscr{A}^{\text {qnil }}$, then $a$ is generalized Drazin invertible and $a^{d}=0$.

In this paper, we first give the formulas of $(a+b)^{d}$ under the conditions $a b=b a b^{\pi}$ and $a b=a^{\pi} b a b^{\pi}$, respectively. Then we will apply these formulas to provide some representations for the generalized Drazin inverse of the operator matrix $M=$ $\left[\begin{array}{ll}A & B \\ C & D\end{array}\right]$ under some conditions. 


\section{Main Results}

First we start the following result which is proved in [8] for matrices, extended in [9] for a bounded linear operator and in [10] for arbitrary elements in a Banach algebra.

Lemma 1 (see [10, Theorem 2.3]). Let $x, y \in \mathscr{A}$ and $p \in \mathscr{A}$ be an idempotent. Assume that $x$ and $y$ are represented as

$$
x=\left[\begin{array}{ll}
a & 0 \\
c & b
\end{array}\right]_{p}, \quad y=\left[\begin{array}{ll}
b & c \\
0 & a
\end{array}\right]_{p} .
$$

(i) If $a \in(p \mathscr{A} p)^{d}$ and $b \in(\bar{p} \mathscr{A} \bar{p})^{d}$, then $x$ and $y$ are generalized Drazin invertible, and

$$
x^{d}=\left[\begin{array}{cc}
a^{d} & 0 \\
u & b^{d}
\end{array}\right]_{p}, \quad y^{d}=\left[\begin{array}{cc}
b^{d} & u \\
0 & a^{d}
\end{array}\right]_{p},
$$

where

$$
u=\sum_{n=0}^{\infty}\left(b^{d}\right)^{n+2} c a^{n} a^{\pi}+\sum_{n=0}^{\infty} b^{\pi} b^{n} c\left(a^{d}\right)^{n+2}-b^{d} c a^{d} .
$$

(ii) If $x \in \mathscr{A}^{d}$ and $a \in(p \mathscr{A} p)^{d}$, then $b \in(\bar{p} \mathscr{A} \bar{p})^{d}$ and $x^{d}$ and $y^{d}$ are given by (6) and (7).

Lemma 2 (see [11, Lemma 2.1]). Let $a, b \in \mathscr{A}^{q n i l}$. If $a b=b a$ or $a b=0$, then $a+b \in \mathscr{A}^{\text {qnil }}$. 3.4].

The following result is a generalization of [10, Corollary

Theorem 3. If $a \in \mathscr{A}^{q n i l}, b \in \mathscr{A}^{d}$, and $a b=b a b^{\pi}$, then $a+b \in$ $\mathscr{A}^{d}$ and

$$
(a+b)^{d}=b^{d}+\sum_{n=0}^{\infty}\left(b^{d}\right)^{n+2} a(a+b)^{n} .
$$

Proof. First, suppose that $b \in \mathscr{A}^{\text {qnil }}$. Therefore, $b^{\pi}=\mathbb{1}$ and from $a b=b a b^{\pi}$ we obtain $a b=b a$. Using Lemma $2, a+b \epsilon$ $\mathscr{A}^{\text {qnil }}$ and (8) holds.

Now we assume $b$ is not quasinilpotent, using matrix representations of $a$ and $b$ relative to $p=b b^{d}$. We have

$$
b=\left[\begin{array}{cc}
b_{1} & 0 \\
0 & b_{2}
\end{array}\right]_{p}, \quad b^{d}=\left[\begin{array}{cc}
b_{1}^{d} & 0 \\
0 & 0
\end{array}\right]_{p}
$$

where $b_{1} \in(p \mathscr{A} p)^{-1}, b_{2} \in(\bar{p} \mathscr{A} \bar{p})^{\text {qnil }}$.

Let us represent

$$
a=\left[\begin{array}{ll}
a_{1} & a_{2} \\
a_{3} & a_{4}
\end{array}\right]_{p}
$$

From $a b=b a b^{\pi}$ and

$$
a b=\left[\begin{array}{ll}
a_{1} b_{1} & a_{2} b_{2} \\
a_{3} b_{1} & a_{4} b_{2}
\end{array}\right]_{p}, \quad b a b^{\pi}=\left[\begin{array}{ll}
0 & b_{1} a_{2} \\
0 & b_{2} a_{4}
\end{array}\right]_{p},
$$

we obtain $a_{1} b_{1}=0$ and $a_{3} b_{1}=0$. Since $b_{1}$ is invertible, we have $a_{1}=0$ and $a_{3}=0$.

Hence we have

$$
a+b=\left[\begin{array}{cc}
b_{1} & a_{2} \\
0 & a_{4}+b_{2}
\end{array}\right]_{p} .
$$

The condition $a b=b a b^{\pi}$ implies that $a_{4} b_{2}=b_{2} a_{4}$. Hence, using Lemma 2 , we get $a_{4}+b_{2} \in \mathscr{A}^{\text {qnil }}$. By Lemma 1 , we obtain that $a+b \in \mathscr{A}^{d}$ and

$$
(a+b)^{d}=\left[\begin{array}{cc}
b_{1}^{d} & u \\
0 & 0
\end{array}\right]_{p},
$$

where

$$
u=\sum_{n=0}^{\infty}\left(b_{1}^{d}\right)^{n+2} a_{2}\left(a_{4}+b_{2}\right)^{n}
$$

Now from (14), using the matrix representation of $b^{d}, a$, and $a+b$, we easily obtain formula (8) of the theorem.

The next result is a generalization of [12, Theorem 2.2] and [10, Example 4.5].

Theorem 4. Let $a, b \in \mathscr{A}^{d}$. If $a b=a^{\pi} b a b^{\pi}$, then $a+b \in \mathscr{A}^{d}$ and

$$
\begin{aligned}
(a+b)^{d}= & b^{\pi} a^{d}+b^{d} a^{\pi}+\sum_{n=0}^{\infty}\left(b^{d}\right)^{n+2} a(a+b)^{n} a^{\pi} \\
& +b^{\pi} \sum_{n=0}^{\infty}(a+b)^{n} b\left(a^{d}\right)^{n+2} \\
& -\sum_{n=0}^{\infty} \sum_{k=0}^{\infty}\left(b^{d}\right)^{k+1} a(a+b)^{n+k} b\left(a^{d}\right)^{n+2} \\
& -\sum_{n=0}^{\infty}\left(b^{d}\right)^{n+2} a(a+b)^{n} b a^{d} .
\end{aligned}
$$

Proof. If $a$ is quasinilpotent, we can apply Theorem 3 and we obtain (15) for this particular case. Now we assume that $a$ is neither invertible nor quasinilpotent and consider the following matrix representations of $a, a^{d}$, and $b$ relative to the $p=a a^{d}$ :

$$
a=\left[\begin{array}{cc}
a_{1} & 0 \\
0 & a_{2}
\end{array}\right]_{p}, \quad a^{d}=\left[\begin{array}{cc}
a_{1}^{d} & 0 \\
0 & 0
\end{array}\right]_{p}, \quad b=\left[\begin{array}{ll}
b_{1} & b_{2} \\
b_{3} & b_{4}
\end{array}\right]_{p} .
$$

The condition $a b=a^{\pi} b a b^{\pi}$ implies that $a_{1} b_{1}=0$ and $a_{1} b_{2}=0$. Since $a_{1}$ is invertible, we have $b_{1}=0$ and $b_{2}=0$.

Thus, $b$ can be represented as

$$
b=\left[\begin{array}{cc}
0 & 0 \\
b_{3} & b_{4}
\end{array}\right]_{p}
$$

Therefore, $b_{4} \in(\bar{p} \mathscr{A} \bar{p})^{d}$ and, from Lemma 1, we have

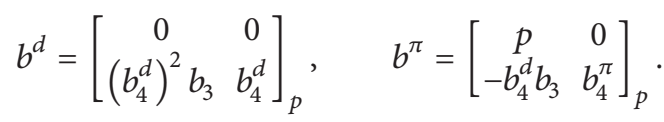


From $a b=a^{\pi} b a b^{\pi}$ and

$$
\begin{gathered}
a b=\left[\begin{array}{cc}
0 & 0 \\
a_{2} b_{3} & a_{2} b_{4}
\end{array}\right]_{p}, \\
a^{\pi} b a b^{\pi}=\left[\begin{array}{cc}
0 & 0 \\
b_{3} a_{1}-b_{4} a_{2} b_{4}^{d} b_{3} & b_{4} a_{2} b_{4}^{\pi}
\end{array}\right]_{p},
\end{gathered}
$$

we obtained $a_{2} b_{4}=b_{4} a_{2} b_{4}^{\pi}$. From Theorem 3, we get $a_{2}+b_{4} \epsilon$ $\mathscr{A}^{d}$ and

$$
\left(a_{2}+b_{4}\right)^{d}=b_{4}^{d}+\sum_{n=0}^{\infty}\left(b_{4}^{d}\right)^{n+2} a_{2}\left(a_{2}+b_{4}\right)^{n} .
$$

Further, applying Lemma 1 to $a+b$, we get

$$
(a+b)^{d}=\left[\begin{array}{cc}
a_{1}^{d} & 0 \\
u & \left(a_{2}+b_{4}\right)^{d}
\end{array}\right]
$$

where

$$
\begin{aligned}
u= & \sum_{n=0}^{\infty}\left[\left(a_{2}+b_{4}\right)^{d}\right]^{n+2} b_{3} a_{1}^{n} a_{1}^{\pi} \\
& +\sum_{n=0}^{\infty}\left(a_{2}+b_{4}\right)^{\pi}\left(a_{2}+b_{4}\right)^{n} b_{3}\left(a_{1}^{d}\right)^{n+2} \\
& -\left(a_{2}+b_{4}\right)^{d} b_{3} a_{1}^{d} .
\end{aligned}
$$

Observe that since $a_{1} \in(p \mathscr{A} p)^{-1}$, then $a_{1}^{\pi}=0$.

Hence, the expression of $u$ reduces to

$$
\begin{aligned}
u= & \sum_{n=0}^{\infty}\left(a_{2}+b_{4}\right)^{\pi}\left(a_{2}+b_{4}\right)^{n} b_{3}\left(a_{1}^{d}\right)^{n+2} \\
& -\left(a_{2}+b_{4}\right)^{d} b_{3} a_{1}^{d} .
\end{aligned}
$$

From $a_{2} b_{4}=b_{4} a_{2} b_{4}^{\pi}$ we get $=a_{2} b_{4}\left(b_{4}^{d}\right)^{2}=b_{4} a_{2} b_{4}^{\pi}\left(b_{4}^{d}\right)^{2}=0$.

Hence, from formula (20) and $a_{2} b_{4}^{d}=0$, we have

$$
\begin{aligned}
\left(a_{2}+b_{4}\right)^{\pi}= & \bar{p}-\left(a_{2}+b_{4}\right) \\
& \cdot\left(b_{4}^{d}+\sum_{n=0}^{\infty}\left(b_{4}^{d}\right)^{n+2} a_{2}\left(a_{2}+b_{4}\right)^{n}\right) \\
= & \bar{p}-b_{4}\left(b_{4}^{d}+\sum_{n=0}^{\infty}\left(b_{4}^{d}\right)^{n+2} a_{2}\left(a_{2}+b_{4}\right)^{n}\right) \\
= & b_{4}^{\pi}-\sum_{n=0}^{\infty}\left(b_{4}^{d}\right)^{n+1} a_{2}\left(a_{2}+b_{4}\right)^{n} .
\end{aligned}
$$

Then substituting (20) and (24) in (22), we get

$$
\begin{aligned}
u= & \sum_{n=0}^{\infty} b_{4}^{\pi}\left(a_{2}+b_{4}\right)^{n} b_{3}\left(a_{1}^{d}\right)^{n+2} \\
& -\sum_{n=0}^{\infty} \sum_{k=0}^{\infty}\left(b_{4}^{d}\right)^{k+1} a_{2}\left(a_{2}+b_{4}\right)^{n+k} b_{3}\left(a_{1}^{d}\right)^{n+2} \\
& -b_{4}^{d} b_{3} a_{1}^{d}-\sum_{n=0}^{\infty}\left(b_{4}^{d}\right)^{n+2} a_{2}\left(a_{2}+b_{4}\right)^{n} b_{3} a_{1}^{d} .
\end{aligned}
$$

Now, replacing $u$ by the above expression and considering matrix representations of $a$ and $b$, after direct computations, we obtain the formula (15) for $(a+b)^{d}$.

\section{Applications}

In this section, we give some formulas for the generalized Drazin inverse of a $2 \times 2$ operator matrix under some conditions.

Finding an explicit representation for the generalized Drazin inverse of an operator matrix $M=\left[\begin{array}{ll}A & B \\ C & D\end{array}\right]$ in terms of $A, B, C, D$ and related generalized Drazin inverse has been studied by several authors [9, 13-15]. Djordjević and Stanimirović [9] generalize the well-known result in $[8,16]$ concerning the Drazin inverse of block $2 \times 2$ upper triangular matrices to the generalized Drazin inverse for a block triangular operator matrix. Further, they consider the case where $B C=0, B D=0$, and $D C=0$.

This section is devoted to the generalized Drazin inverse of $2 \times 2$ operator matrix:

$$
M=\left[\begin{array}{ll}
A & B \\
C & D
\end{array}\right]
$$

where $A \in \mathbf{B}(X)$ and $D \in \mathbf{B}(Y)$ are generalized Drazin invertible.

Next we will state some auxiliary lemmas.

Lemma 5 (see $[2,3])$. Let $A$ and $D$ be generalized Drazin invertible and let $M$ be matrix of form (26). If $B C=0$ and $B D=0$, then

$$
M^{d}=\left[\begin{array}{cc}
A^{d} & \left(A^{d}\right)^{2} B \\
X_{0} & D^{d}+X_{1} B
\end{array}\right]
$$

where

$$
\begin{aligned}
X_{n}= & \sum_{i=0}^{\infty}\left(D^{d}\right)^{i+n+2} C A^{i} A^{\pi} \\
& +D^{\pi} \sum_{i=0}^{\infty} D^{i} C\left(A^{d}\right)^{i+n+2} \\
& -\sum_{i=0}^{n}\left(D^{d}\right)^{i+1} C\left(A^{d}\right)^{n-i+1}, \quad n \geq 0 .
\end{aligned}
$$

Lemma 6 (see [17, Lemma 3.1]). If $M$ is matrix of form (26), such that $A$ is generalized Drazin invertible, $D$ is quasinilpotent, and $B D^{n} C=0$ for any nonnegative integer $n$, then $M$ is generalized Drazin invertible and

$$
M^{d}=\left[\begin{array}{cc}
A^{d} & \Gamma \\
\Delta & \Delta A \Gamma
\end{array}\right]
$$

where

$$
\Gamma=\sum_{n=0}^{\infty}\left(A^{d}\right)^{n+2} B D^{n}, \quad \Delta=\sum_{n=0}^{\infty} D^{n} C\left(A^{d}\right)^{n+2} .
$$


Lemma 7. Let $A \in \mathbb{C}^{n \times n}$. Then $\left(A A^{\pi}\right)^{d}=0,\left(A^{2} A^{d}\right)^{d}=A^{d}$, $\left(A^{2} A^{d}\right)^{\pi}=A^{\pi}$, and $\operatorname{Ind}\left(A A^{\pi}\right)=\operatorname{Ind}(A)$ and $\operatorname{Ind}\left(A^{2} A^{d}\right)=1$.

Proof. The Jordan canonical form of $X$ permits us to write $A=S(C \oplus N) S^{-1}$, where $S$ and $C$ are nonsingular, and $N$ is nilpotent with index $\operatorname{Ind}(A)$. Thus $A_{d}=S\left(C^{-1} \oplus 0\right) S^{-1}$. Now, it is evident that $A^{2} A^{d}=S(C \oplus 0) S^{-1}$ and $A A^{\pi}=S(0 \oplus N) S^{-1}$, which lead to the affirmations of this lemma.

In [9, Theorem 5.3] authors gave an explicit representation for $M^{d}$ under conditions $B C=0, D C=0$, and $B D=0$. Here we replace the last two conditions by the two weaker conditions $D C=D^{\pi} C A A^{\pi}$ and $B D=A A^{\pi} B$.

Theorem 8. Let $A$ and $D$ be generalized Drazin invertible and let $M$ be matrix of form (26). If $A A^{\pi} B=B D, D C=D^{\pi} C A A^{\pi}$ and $B C=0$. Then

$$
M^{d}=\left[\begin{array}{cc}
A^{d} & \left(A^{d}\right)^{2} B+\sum_{n=0}^{\infty} A^{n} B\left(D^{d}\right)^{n+2} \\
C\left(A^{d}\right)^{2} & D^{d}+C\left(A^{d}\right)^{3} B+\sum_{n=1}^{\infty} \sum_{i=1}^{n} D^{i-1} C A^{n-i} B\left(D^{d}\right)^{n+2}
\end{array}\right] .
$$

Proof. We can split matrix $M$ as $M=P+Q$, where

$$
\begin{array}{cc}
P=\left[\begin{array}{cc}
A A^{\pi} & 0 \\
0 & D
\end{array}\right], & Q=\left[\begin{array}{cc}
A^{2} A^{d} & B \\
C & 0
\end{array}\right], \\
P^{d}=\left[\begin{array}{cc}
0 & 0 \\
0 & D^{d}
\end{array}\right], & P^{\pi}=\left[\begin{array}{cc}
I & 0 \\
0 & D^{\pi}
\end{array}\right] .
\end{array}
$$

Since $D C=D^{\pi} C A A^{\pi}$ and $A A^{\pi} B=B D$, we have

$$
\begin{gathered}
D^{d} C=\left(D^{d}\right)^{2} D C=\left(D^{d}\right)^{2} D^{\pi} C A A^{\pi}=0, \\
D C A^{d}=D^{\pi} C A A^{\pi} A^{d}=0 \\
A^{d} B D=A^{d} A A^{\pi} B=0 .
\end{gathered}
$$

From $B C=0$ and applying Lemma 5 to $Q$, we obtain

$$
\begin{gathered}
\left(Q^{d}\right)^{i}=\left[\begin{array}{cc}
\left(A^{d}\right)^{i} & \left(A^{d}\right)^{i+1} B \\
X_{i-1} & X_{i} B
\end{array}\right], \\
Q^{\pi}=\left[\begin{array}{cc}
A^{\pi} & -A^{d} B \\
-C A^{d} & I-C\left(A^{d}\right)^{2} B
\end{array}\right],
\end{gathered}
$$

where $X_{n}$ is defined in (28). From $D^{d} C=0$ and $D C A^{d}=0$, we get $X_{n}=C\left(A^{d}\right)^{n+2}$.

Since $A A^{\pi} B=B D$ and $D C=D^{\pi} C A A^{\pi}$, we obtain $P Q=$ $P^{\pi} Q P Q^{\pi}$. Applying Theorem 4 , we get

$$
\begin{aligned}
(P+Q)^{d}= & Q^{\pi} P^{d}+Q^{d} P^{\pi} \\
& +\sum_{n=0}^{\infty}\left(Q^{d}\right)^{n+2} P(P+Q)^{n} P^{\pi}
\end{aligned}
$$

$$
\begin{aligned}
& +Q^{\pi} \sum_{n=0}^{\infty}(P+Q)^{n} Q\left(P^{d}\right)^{n+2} \\
& -\sum_{n=0}^{\infty} \sum_{k=0}^{\infty}\left(Q^{d}\right)^{k+1} P(P+Q)^{n+k} Q\left(P^{d}\right)^{n+2} \\
& -\sum_{n=0}^{\infty}\left(Q^{d}\right)^{n+2} P(P+Q)^{n} Q P^{d}
\end{aligned}
$$

From $A^{d} B D=0$, we have

$$
Q^{\pi} P^{d}=P^{d}, \quad Q^{d} P^{\pi}=Q^{d}, \quad Q^{d} P=0 .
$$

Hence from (35), we obtain

$$
(P+Q)^{d}=P^{d}+Q^{d}+Q^{\pi} \sum_{n=0}^{\infty}(P+Q)^{n} Q\left(P^{d}\right)^{n+2}
$$

Since $A^{d} B D=0$, we have

$$
\mathrm{Q}^{\pi} \mathrm{Q}\left(P^{d}\right)^{2}=B\left(D^{d}\right)^{2} \text {. }
$$

The conditions $B C=0$ and $B D=A A^{\pi} B$ imply that $B D^{n} C=0$. From $B D^{n} C=0$ and $A^{d} B D=0$, we get

$$
\begin{aligned}
& Q^{\pi} \sum_{n=1}^{\infty}(P+Q)^{n} Q\left(P^{d}\right)^{n+2} \\
& =\left[\begin{array}{cc}
0 & \sum_{n=1}^{\infty} A^{n} B\left(D^{d}\right)^{n+2} \\
0 & \sum_{n=1}^{\infty} \sum_{i=1}^{n} D^{i-1} C A^{n-i} B\left(D^{d}\right)^{n+2}
\end{array}\right] .
\end{aligned}
$$

From (36), (38), and (39) it follows (31).

The proof is finished.

Since

$$
\left[\begin{array}{ll}
A & B \\
C & D
\end{array}\right]=\left[\begin{array}{cc}
0 & I_{n} \\
I_{m} & 0
\end{array}\right]\left[\begin{array}{cc}
D & C \\
B & A
\end{array}\right]\left[\begin{array}{cc}
0 & I_{m} \\
I_{n} & 0
\end{array}\right]
$$

we can obtain the following result, applying Theorem 8 to $\left[\begin{array}{ll}D & C \\ B & A\end{array}\right]$.

Theorem 9. Let $A$ and $D$ be generalized Drazin invertible and let $M$ be matrix of form (26). If $D D^{\pi} C=C A, A B=A^{\pi} B D D^{\pi}$ and $C B=0$. Then

$$
M^{d}=\left[\begin{array}{cc}
A^{d}+B\left(D^{d}\right)^{3} C+\sum_{n=1}^{\infty} \sum_{i=1}^{n} A^{n-i} B D^{i-1} C\left(A^{d}\right)^{n+2} & B\left(D^{d}\right)^{2} \\
\left(D^{d}\right)^{2} C+\sum_{n=0}^{\infty} D^{n} C\left(A^{d}\right)^{n+2} & D^{d}
\end{array}\right] .
$$

Theorem 10. Let $A, D$, and $B C$ be generalized Drazin invertible and let $M$ be matrix of form (26). If $A B=A^{\pi} B D, D C=$ $D^{\pi} C A$ and $B C=0$. Then

$$
M^{d}=\left[\begin{array}{cc}
A^{d} & \sum_{n=0}^{\infty} A^{n} B\left(D^{d}\right)^{n+2} \\
\sum_{n=0}^{\infty} D^{n} C\left(A^{d}\right)^{n+2} & D^{d}+\sum_{n=1}^{\infty} \sum_{i=0}^{n-1} D^{i} C A^{n-i-1} B\left(D^{d}\right)^{n+2}
\end{array}\right]
$$


Proof. We can split matrix $M$ as $M=P+Q$, where

$$
\begin{array}{cc}
P=\left[\begin{array}{cc}
A & 0 \\
0 & D
\end{array}\right], & Q=\left[\begin{array}{cc}
0 & B \\
C & 0
\end{array}\right], \\
P^{d}=\left[\begin{array}{cc}
A^{d} & 0 \\
0 & D^{d}
\end{array}\right], & P^{\pi}=\left[\begin{array}{cc}
A^{\pi} & 0 \\
0 & D^{\pi}
\end{array}\right] .
\end{array}
$$

Since

$$
Q^{2}=\left[\begin{array}{cc}
B C & 0 \\
0 & C B
\end{array}\right], \quad Q^{3}=\left[\begin{array}{cc}
0 & B C B \\
C B C & 0
\end{array}\right]
$$

from $B C=0$, it is easy to get $Q^{3}=0$. Since $Q$ is nilpotent, we have $Q^{d}=0$. Applying Theorem 4 to the particular case, we get

$$
(P+Q)^{d}=P^{d}+\sum_{n=0}^{\infty}(P+Q)^{n} Q\left(P^{d}\right)^{n+2}
$$

The conditions $A B=A^{\pi} B D$ and $B C=0$ imply that $B D^{n} C=0$, for $n \geq 0$, so we get

$$
\begin{aligned}
\sum_{n=0}^{\infty}(P+Q)^{n} Q\left(P^{d}\right)^{n+2} & \\
& =\left[\begin{array}{cc}
0 & \sum_{n=0}^{\infty} A^{n} B\left(D^{d}\right)^{n+2} \\
\sum_{n=0}^{\infty} D^{n} C\left(A^{d}\right)^{n+2} & \sum_{n=1}^{\infty} \sum_{i=0}^{n-1} D^{i} C A^{n-i-1} B\left(D^{d}\right)^{n+2}
\end{array}\right] .
\end{aligned}
$$

From (44) and (47) it follows (42).

The proof is finished.

Theorem 11. Let $A$ and $D$ be generalized Drazin invertible and let $M$ be matrix of form (26). If $A A^{\pi} B=B D^{2} D^{d}, D^{2} D^{d} C=$ $D^{\pi} C A A^{\pi}$ and $B D^{n} C=0$ for any nonnegative integer $n$. Then

$$
M^{d}=\left[\begin{array}{cc}
A^{d} & \Gamma+\sum_{n=0}^{\infty} A^{n} B\left(D^{d}\right)^{n+2} \\
\Delta & D^{d}+\Delta A \Gamma+\sum_{n=1}^{\infty} \sum_{i=0}^{n-1} D^{i} C A^{n-i-1} B\left(D^{d}\right)^{n+2}
\end{array}\right],
$$

where $\Gamma$ and $\Delta$ are defined in (30).

Proof. We can split matrix $M$ as $M=P+Q$, where

$$
\begin{array}{cc}
P=\left[\begin{array}{cc}
A A^{\pi} & 0 \\
0 & D^{2} D^{d}
\end{array}\right], & Q=\left[\begin{array}{cc}
A^{2} A^{d} & B \\
C & D D^{\pi}
\end{array}\right], \\
P^{d}=\left[\begin{array}{cc}
0 & 0 \\
0 & D^{d}
\end{array}\right], & P^{\pi}=\left[\begin{array}{cc}
I & 0 \\
0 & D^{\pi}
\end{array}\right] .
\end{array}
$$

From $A A^{\pi} B=B D^{2} D^{d}$ and $D^{2} D^{d} C=D^{\pi} C A A^{\pi}$, we have

$$
\begin{aligned}
D^{d} C & =\left(D^{d}\right)^{3} D^{2} C=\left(D^{d}\right)^{2} D^{2} D^{d} C \\
& =\left(D^{d}\right)^{2} D^{\pi} C A A^{\pi}=0, \\
A^{d} B D^{d} & =A^{d} B D^{2}\left(D^{d}\right)^{3}=A^{d} B D^{2} D^{d}\left(D^{d}\right)^{2} \\
& =A^{d} A A^{\pi} B\left(D^{d}\right)^{2}=0,
\end{aligned}
$$

so we get $D^{\pi} C=C$.

Note that $D D^{\pi}$ is quasinilpotent, $D^{\pi} C=C$, and $B\left(D D^{\pi}\right)^{n} C=B D^{n} D^{\pi} C=B D^{n} C=0$ for any nonnegative integer $n$; we can apply Lemma 6 to $Q$ with $D$ replaced by $D D^{\pi}$; we have

$$
Q^{d}=\left[\begin{array}{cc}
A^{d} & \Gamma^{\prime} \\
\Delta^{\prime} & \Delta^{\prime} A \Gamma^{\prime}
\end{array}\right]
$$

where

$$
\Gamma^{\prime}=\sum_{n=0}^{\infty}\left(A^{d}\right)^{n+2} B D^{n} D^{\pi}, \quad \Delta^{\prime}=\sum_{n=0}^{\infty} D^{n} D^{\pi} C\left(A^{d}\right)^{n+2}
$$

Observe that (50) and (51) yield

$$
\Gamma=\sum_{n=0}^{\infty}\left(A^{d}\right)^{n+2} B D^{n}, \quad \Delta=\sum_{n=0}^{\infty} D^{n} C\left(A^{d}\right)^{n+2},
$$

so we get

$$
Q^{d}=\left[\begin{array}{cc}
A^{d} & \Gamma \\
\Delta & \Delta A \Gamma
\end{array}\right]
$$

The condition $B D^{n} C=0$ implies that

$$
B \Delta=B \sum_{n=0}^{\infty} D^{n} C\left(A^{d}\right)^{n+2}=0
$$

Hence we have

$$
\begin{aligned}
Q Q^{d} & =\left[\begin{array}{cc}
A A^{d}+B \Delta & A^{2} A^{d} \Gamma+B \Delta A \Gamma \\
C A^{d}+D D^{\pi} \Delta & C \Gamma+D D^{\pi} \Delta A \Gamma
\end{array}\right] \\
& =\left[\begin{array}{cc}
A A^{d} & A \Gamma \\
C A^{d}+D \Delta & C \Gamma+D \Delta A \Gamma
\end{array}\right], \\
Q^{\pi} & =\left[\begin{array}{cc}
A^{\pi} & -A \Gamma \\
-C A^{d}-D \Delta & I-C \Gamma-D \Delta A \Gamma
\end{array}\right] .
\end{aligned}
$$


From $A A^{\pi} B=B D^{2} D^{d}$ and $D^{2} D^{d} C=D^{\pi} C A A^{\pi}$, we obtain $P Q=P^{\pi} Q P Q^{\pi}$. Applying Theorem 4, we get

$$
\begin{aligned}
(P+Q)^{d}= & Q^{\pi} P^{d}+Q^{d} P^{\pi} \\
& +\sum_{n=0}^{\infty}\left(Q^{d}\right)^{n+2} P(P+Q)^{n} P^{\pi} \\
& +Q^{\pi} \sum_{n=0}^{\infty}(P+Q)^{n} Q\left(P^{d}\right)^{n+2} \\
& -\sum_{n=0}^{\infty} \sum_{k=0}^{\infty}\left(Q^{d}\right)^{k+1} P(P+Q)^{n+k} Q\left(P^{d}\right)^{n+2} \\
& -\sum_{n=0}^{\infty}\left(Q^{d}\right)^{n+2} P(P+Q)^{n} Q P^{d},
\end{aligned}
$$

$$
\Delta A A^{\pi}=\sum_{n=0}^{\infty} D^{n} D^{\pi} C\left(A^{d}\right)^{n+2} A A^{\pi}=0
$$

so we get

$$
\begin{aligned}
Q^{d} P & =\left[\begin{array}{cc}
A^{d} & \Gamma \\
\Delta & \Delta A \Gamma
\end{array}\right]\left[\begin{array}{cc}
A A^{\pi} & 0 \\
0 & D^{2} D^{d}
\end{array}\right] \\
& =\left[\begin{array}{cc}
A^{d} A A^{\pi} & \Gamma D^{2} D^{d} \\
\Delta A A^{\pi} & \Delta A \Gamma D^{2} D^{d}
\end{array}\right]=\left[\begin{array}{ll}
0 & 0 \\
0 & 0
\end{array}\right] .
\end{aligned}
$$

Hence from (58) and (60) we obtain

$$
\begin{aligned}
(P+Q)^{d}= & Q^{\pi} P^{d}+Q^{d} P^{\pi} \\
& +Q^{\pi} \sum_{n=0}^{\infty}(P+Q)^{n} Q\left(P^{d}\right)^{n+2} .
\end{aligned}
$$

By direct computation we verify that

$$
Q^{\pi} P^{d}=P^{d}, \quad Q^{d} P^{\pi}=Q^{d} .
$$

From $B D^{n} C=0$, we have

$$
\begin{aligned}
\sum_{n=0}^{\infty}(P+Q)^{n} Q\left(P^{d}\right)^{n+2} & \\
& =\left[\begin{array}{cc}
0 & \sum_{n=0}^{\infty} A^{n} B\left(D^{d}\right)^{n+2} \\
0 & \sum_{n=1}^{\infty} \sum_{i=0}^{n-1} D^{i} C A^{n-i-1} B\left(D^{d}\right)^{n+2}
\end{array}\right] .
\end{aligned}
$$

Observe that (51) and $B D^{n} C=0$ yield

$$
Q^{\pi} \sum_{n=0}^{\infty}(P+Q)^{n} Q\left(P^{d}\right)^{n+2}
$$$$
\begin{aligned}
& =\left[\begin{array}{cc}
A^{\pi} & -A \Gamma \\
-C A^{d}-D \Delta & I-C \Gamma-D \Delta A \Gamma
\end{array}\right]\left[\begin{array}{cc}
0 & \sum_{n=0}^{\infty} A^{n} B\left(D^{d}\right)^{n+2} \\
0 & \sum_{n=1}^{\infty} \sum_{i=0}^{n-1} D^{i} C A^{n-i-1} B\left(D^{d}\right)^{n+2}
\end{array}\right] \\
& =\left[\begin{array}{cc}
0 & A^{\pi} \sum_{n=0}^{\infty} A^{n} B\left(D^{d}\right)^{n+2}-A \Gamma \sum_{n=1}^{\infty} \sum_{i=0}^{n-1} D^{i} C A^{n-i-1} B\left(D^{d}\right)^{n+2} \\
0 & \left(-C A^{d}-D \Delta\right) \sum_{n=0}^{\infty} A^{n} B\left(D^{d}\right)^{n+2}+(I-C \Gamma-D \Delta A \Gamma) \sum_{n=1}^{\infty} \sum_{i=0}^{n-1} D^{i} C A^{n-i-1} B\left(D^{d}\right)^{n+2}
\end{array}\right] \\
& =\left[\begin{array}{cc}
0 & \sum_{n=0}^{\infty} A^{n} B\left(D^{d}\right)^{n+2} \\
0 & \sum_{n=1}^{\infty} \sum_{i=0}^{n-1} D^{i} C A^{n-i-1} B\left(D^{d}\right)^{n+2}
\end{array}\right] .
\end{aligned}
$$ 
Theorem 12. Let $A$ and $D$ be generalized Drazin invertible and let $M$ be matrix of form (26). If $A A^{\pi} B D^{\pi}=B D, B C=0$, $C A^{d}=0$, and $C B D^{\pi}=0$. Then

$$
M^{d}=\left[\begin{array}{cc}
A^{d} & \left(A^{d}\right)^{2} B \\
X_{0} & D^{d}+\sum_{n=0}^{\infty} X_{n+1} B D^{n}
\end{array}\right],
$$

where

$$
X_{n}=\sum_{i=0}^{\infty}\left(D^{d}\right)^{i+n+2} C A^{i}, \quad n \geq 0 .
$$

Proof. We can split matrix $M$ as $M=P+Q$, where

$$
\begin{array}{cc}
P=\left[\begin{array}{cc}
A^{2} A^{d} & B \\
0 & 0
\end{array}\right], & Q=\left[\begin{array}{cc}
A A^{\pi} & 0 \\
C & D
\end{array}\right], \\
P^{d}=\left[\begin{array}{cc}
A^{d}\left(A^{d}\right)^{2} B \\
0 & 0
\end{array}\right], & P^{\pi}=\left[\begin{array}{cc}
A^{\pi} & -A^{d} B \\
0 & I
\end{array}\right] .
\end{array}
$$

Applying Lemma 7, we have $\left(A A^{\pi}\right)^{d}=0$, so we get

$$
\left(Q^{d}\right)^{n}=\left[\begin{array}{cc}
0 & 0 \\
X_{n-1} & \left(D^{d}\right)^{n}
\end{array}\right], \quad Q^{\pi}=\left[\begin{array}{cc}
I & 0 \\
-D X_{0} & D^{\pi}
\end{array}\right],
$$

where $X_{n}$ is defined in (28).

Since $A A^{\pi} B D^{\pi}=B D, B C=0, C B D^{\pi}=0$, and $C A^{2} A^{d}=$ 0 , we obtain $P Q=P^{\pi} Q P Q^{\pi}$. Applying Theorem 4, we get

$$
\begin{aligned}
(P+Q)^{d}= & Q^{\pi} P^{d}+Q^{d} P^{\pi} \\
& +\sum_{n=0}^{\infty}\left(Q^{d}\right)^{n+2} P(P+Q)^{n} P^{\pi} \\
& +Q^{\pi} \sum_{n=0}^{\infty}(P+Q)^{n} Q\left(P^{d}\right)^{n+2} \\
& -\sum_{n=0}^{\infty} \sum_{k=0}^{\infty}\left(Q^{d}\right)^{k+1} P(P+Q)^{n+k} Q\left(P^{d}\right)^{n+2} \\
& -\sum_{n=0}^{\infty}\left(Q^{d}\right)^{n+2} P(P+Q)^{n} Q P^{d} .
\end{aligned}
$$

From $C A^{d}=0$, we have $Q P^{d}=0$. Hence from (69) we obtain

$$
(P+Q)^{d}=Q^{\pi} P^{d}+Q^{d} P^{\pi}+\sum_{n=0}^{\infty}\left(Q^{d}\right)^{n+2} P(P+Q)^{n} P^{\pi},
$$

where $X_{n} A^{d}=0$, we get

$$
\begin{aligned}
& Q^{\pi} P^{d}=P^{d}, \quad Q^{d} P^{\pi}=Q^{d}, \\
\left(Q^{d}\right)^{2} P P^{\pi} & =\left[\begin{array}{cc}
0 & 0 \\
X_{1} & \left(D^{d}\right)^{2}
\end{array}\right]\left[\begin{array}{cc}
A^{2} A^{d} & B \\
0 & 0
\end{array}\right]\left[\begin{array}{cc}
A^{\pi} & -A^{d} B \\
0 & I
\end{array}\right] \\
& =\left[\begin{array}{cc}
0 & 0 \\
0 & X_{1} B
\end{array}\right] .
\end{aligned}
$$

The conditions $A A^{\pi} B D^{\pi}=B D$ and $B C=0$ imply that $B D^{i} C=0$. So we get

$$
\begin{aligned}
& \sum_{n=0}^{\infty}\left(Q^{d}\right)^{n+2} P(P+Q)^{n} P^{\pi} \\
&=\left[\begin{array}{cc}
0 & 0 \\
0 & \sum_{n=1}^{\infty} X_{n+1} B D^{n}
\end{array}\right], \quad n \geq 1 .
\end{aligned}
$$

From (71) and (72) it follows (65).

The proof is finished.

Using (40) and Theorem 12, we have the following result.

Theorem 13. If $C A=D^{\pi} D C A^{\pi}, B D^{d}=0, C B=0$, and $B C A^{\pi}=0$. Then

$$
M^{d}=\left[\begin{array}{cc}
A^{d}+\sum_{n=0}^{\infty} X_{n+2} C A^{n} & X_{1} \\
\left(D^{d}\right)^{2} C & D^{d}
\end{array}\right],
$$

where

$$
X_{n}=\sum_{i=0}^{\infty}\left(A^{d}\right)^{i+n+1} B D^{i}, \quad n \geq 1 .
$$

Using the case of Theorem 3, we get the following results.

Theorem 14. Let $A$ and $D$ be generalized Drazin invertible and let $M$ be matrix of form (26). If $D C A^{\pi}=C A, A B D=0$, $B C=0$, and $C B=0$. Then

$$
M^{d}=\left[\begin{array}{cc}
A^{d}+\sum_{n=0}^{\infty} B\left(D^{d}\right)^{n+3} C A^{n} & \left(A^{d}\right)^{2} B+B\left(D^{d}\right)^{2} \\
\sum_{n=0}^{\infty}\left(D^{d}\right)^{n+2} C A^{n} & D^{d}
\end{array}\right] .
$$

Proof. We can split matrix $M$ as $M=P+Q$, where

$$
P=\left[\begin{array}{ll}
0 & 0 \\
C & 0
\end{array}\right], \quad Q=\left[\begin{array}{cc}
A & B \\
0 & D
\end{array}\right] .
$$

From $A B D=0$, we have

$$
\begin{gathered}
\left(Q^{d}\right)^{i}=\left[\begin{array}{cc}
\left(A^{d}\right)^{i} & X_{i} \\
0 & \left(D^{d}\right)^{i}
\end{array}\right], \\
Q^{\pi}=\left[\begin{array}{cc}
A^{\pi} & -A X_{1}-B D^{d} \\
0 & D^{\pi}
\end{array}\right],
\end{gathered}
$$

where

$$
X_{n}=\left(A^{d}\right)^{n+2} B+B\left(D^{d}\right)^{n+2}, \quad n \geq 0 .
$$

Note that $P$ is quasinilpotent; since $D C A^{\pi}=C A, A B D=$ $0, B C=0$, and $C B=0$, we obtain $P Q=Q P Q^{\pi}$. Applying Theorem 3, we get

$$
(P+Q)^{d}=Q^{d}+\sum_{n=0}^{\infty}\left(Q^{d}\right)^{n+2} P(P+Q)^{n} .
$$


From $B C=0$, we have

$$
\begin{aligned}
\left(Q^{d}\right)^{2} P & =\left[\begin{array}{cc}
\left(A^{d}\right)^{2} & X_{2} \\
0 & \left(D^{d}\right)^{2}
\end{array}\right]\left[\begin{array}{ll}
0 & 0 \\
C & 0
\end{array}\right] \\
& =\left[\begin{array}{cc}
X_{2} C & 0 \\
\left(D^{d}\right)^{2} C & 0
\end{array}\right]=\left[\begin{array}{ll}
B\left(D^{d}\right)^{3} & 0 \\
\left(D^{d}\right)^{2} C & 0
\end{array}\right] .
\end{aligned}
$$

The conditions $D C A^{\pi}=C A$ and $C B=0$ imply that $C A^{i} B=0$. From $A B D=0, C A^{i} B=0$, and $B C=0$, we get

$$
\begin{aligned}
\sum_{n=1}^{\infty}\left(Q^{d}\right)^{n+2} P(P+Q)^{n} & \\
= & {\left[\begin{array}{cc}
\sum_{n=1}^{\infty} X_{n+2} C A^{n} & \sum_{n=1}^{\infty} X_{n+2} C A^{n-1} B \\
\sum_{n=1}^{\infty}\left(D^{d}\right)^{n+2} C A^{n} & \sum_{n=1}^{\infty}\left(D^{d}\right)^{n+2} C A^{n-1} B
\end{array}\right] } \\
= & {\left[\begin{array}{cc}
\sum_{n=1}^{\infty} B\left(D^{d}\right)^{n+3} C A^{n} & 0 \\
\sum_{n=1}^{\infty}\left(D^{d}\right)^{n+2} C A^{n} & 0
\end{array}\right] . }
\end{aligned}
$$

From (77), (80), and (81) it follows (75).

Using (40) and Theorem 14, we have the following result.

Theorem 15. Let $A$ and $D$ be generalized Drazin invertible and let $M$ be matrix of form (26). If $A B D^{\pi}=B D, D C A=0$, $B C=A B C A^{d}$, and $C B=0$. Then

$$
M^{d}=\left[\begin{array}{cc}
A^{d} & \sum_{n=0}^{\infty}\left(A^{d}\right)^{n+2} B D^{n} \\
\left(D^{d}\right)^{2} C+C\left(A^{d}\right)^{2} & D^{d}+\sum_{n=0}^{\infty} C\left(A^{d}\right)^{n+3} B D^{n}
\end{array}\right] .
$$

\section{Conflict of Interests}

The authors declare that there is no conflict of interests regarding the publication of this paper.

\section{References}

[1] J. J. Koliha, "A generalized Drazin inverse," Glasgow Mathematical Journal, vol. 38, no. 3, pp. 367-381, 1991.

[2] C. Deng, D. S. Cvetković-Ilić, and Y. Wei, "Some results on the generalized Drazin inverse of operator matrices," Linear and Multilinear Algebra, vol. 58, no. 3-4, pp. 503-521, 2010.

[3] E. Dopazo and M. F. Martínez-Serrano, "Further results on the representation of the Drazin inverse of a $2 \times 2$ block matrix," Linear Algebra and its Applications, vol. 432, no. 8, pp. 18961904, 2010.

[4] J. J. Koliha and V. Rakocević, "Holomorphic and meromorphic properties of the $g$-Drazin inverse," Demonstratio Mathematica, vol. 38 , no. 3, pp. 657-666, 2005.
[5] N. Castro González, "Additive perturbation results for the Drazin inverse," Linear Algebra and Its Applications, vol. 397, pp. 279-297, 2005.

[6] X. Liu, L. Xu, and Y. Yu, "The representations of the Drazin inverse of differences of two matrices," Applied Mathematics and Computation, vol. 216, no. 12, pp. 3652-3661, 2010.

[7] D. S. Djordjević and V. Rakočević, Lectures on Generalized Inverses, University of Niš, 2008.

[8] J. Meyer Jr. and N. J. Rose, "The index and the Drazin inverse of block triangular matrices," SIAM Journal on Applied Mathematics, vol. 33, no. 1, pp. 1-7, 1977.

[9] D. S. Djordjević and P. S. Stanimirović, "On the generalized Drazin inverse and generalized resolvent," Czechoslovak Mathematical Journal, vol. 51, no. 3, pp. 617-634, 2001.

[10] N. Castro-González and J. J. Koliha, "New additive results for the $g$-Drazin inverse," Proceedings of the Royal Society of Edinburgh: Section A. Mathematics, vol. 134, no. 6, pp. 10851097, 2004.

[11] D. S. Cvetković-Ilić, D. S. Djordjević, and Y. Wei, "Additive results for the generalized Drazin inverse in a Banach algebra," Linear Algebra and Its Applications, vol. 418, no. 1, pp. 53-61, 2006.

[12] D. S. Djordjević and Y. Wei, "Additive results for the generalized Drazin inverse," Journal of the Australian Mathematical Society, vol. 73, no. 1, pp. 115-125, 2002.

[13] C. Deng and Y. Wei, "A note on the Drazin inverse of an antitriangular matrix," Linear Algebra and its Applications, vol. 431, no. 10, pp. 1910-1922, 2009.

[14] N. Castro-González, E. Dopazo, and M. F. Martínez-Serrano, "On the Drazin inverse of the sum of two operators and its application to operator matrices," Journal of Mathematical Analysis and Applications, vol. 350, no. 1, pp. 207-215, 2009.

[15] R. E. Hartwig, G. Wang, and Y. Wei, "Some additive results on Drazin inverse," Linear Algebra and its Applications, vol. 322, no. 1-3, pp. 207-217, 2001.

[16] R. E. Hartwig and J. Shoaf, "Group inverses and Drazin inverses of bidiagonal and triangular Toeplitz matrices," vol. 24, no. 1, pp. 10-34, 1977.

[17] R. E. Hartwig and J. M. Shoaf, "Group inverse and Drazin inverse of bidiagonal and triangular Toeplitz matrices," Journal of the Australian Mathematical Society A, vol. 24, pp. 10-34, 1977. 


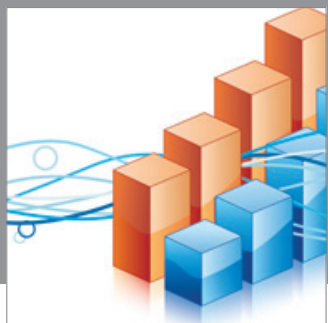

Advances in

Operations Research

mansans

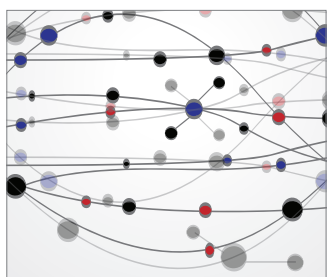

The Scientific World Journal
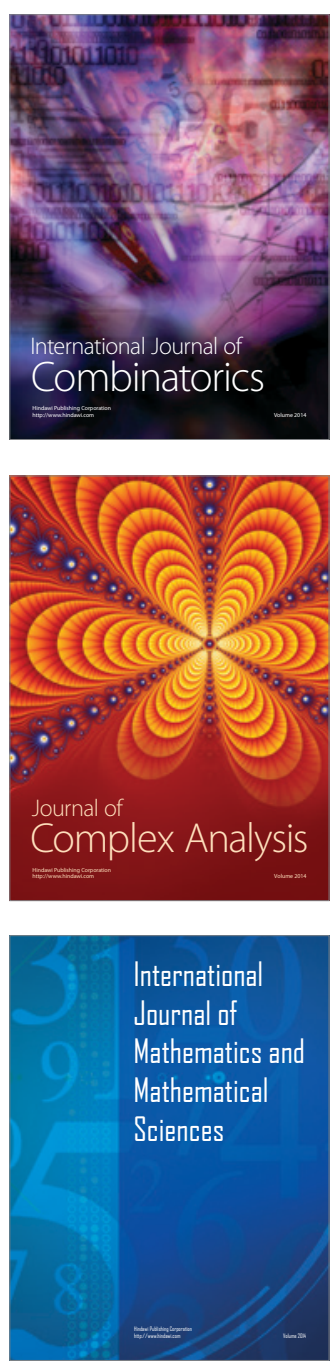
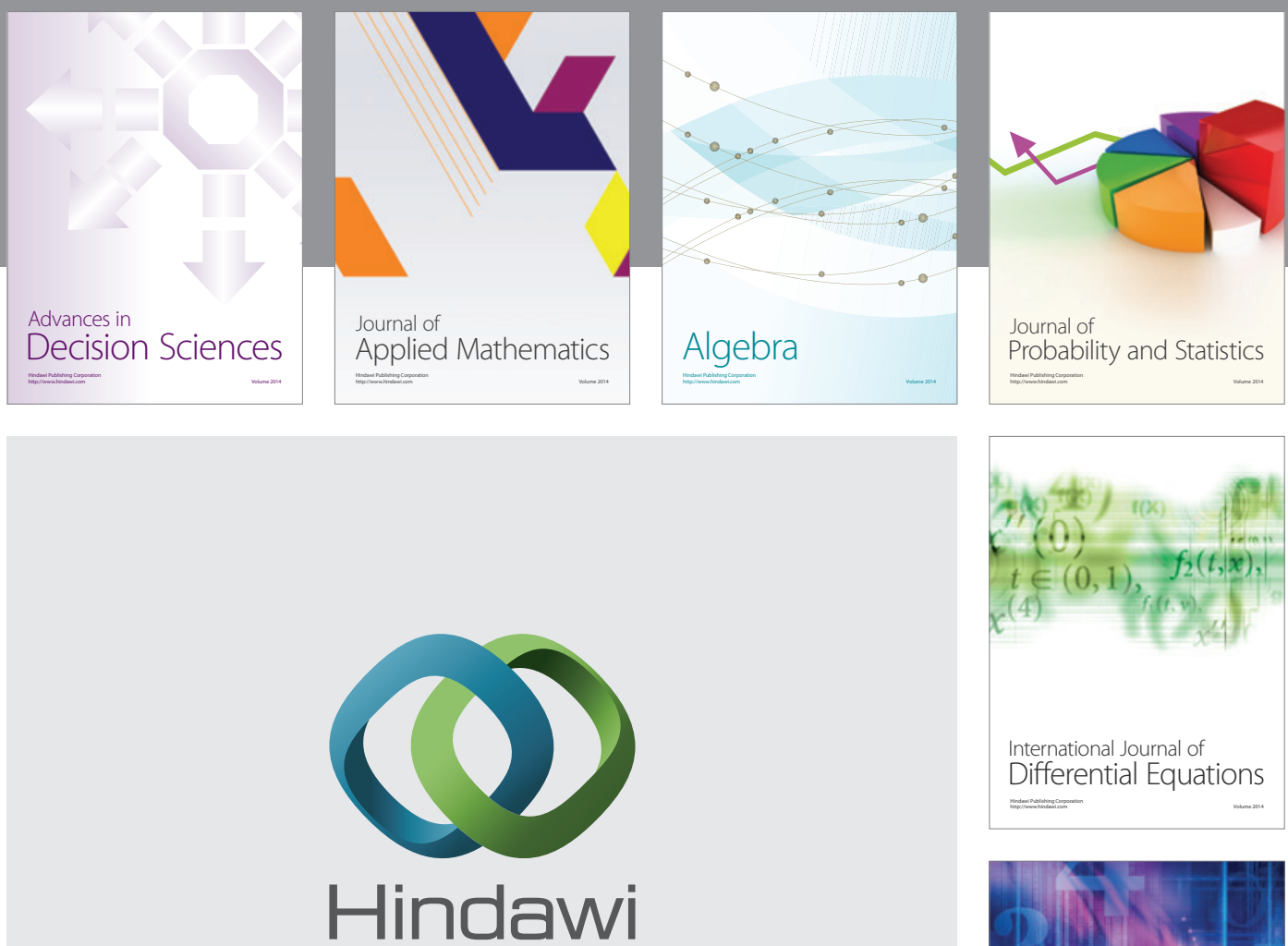

Submit your manuscripts at http://www.hindawi.com
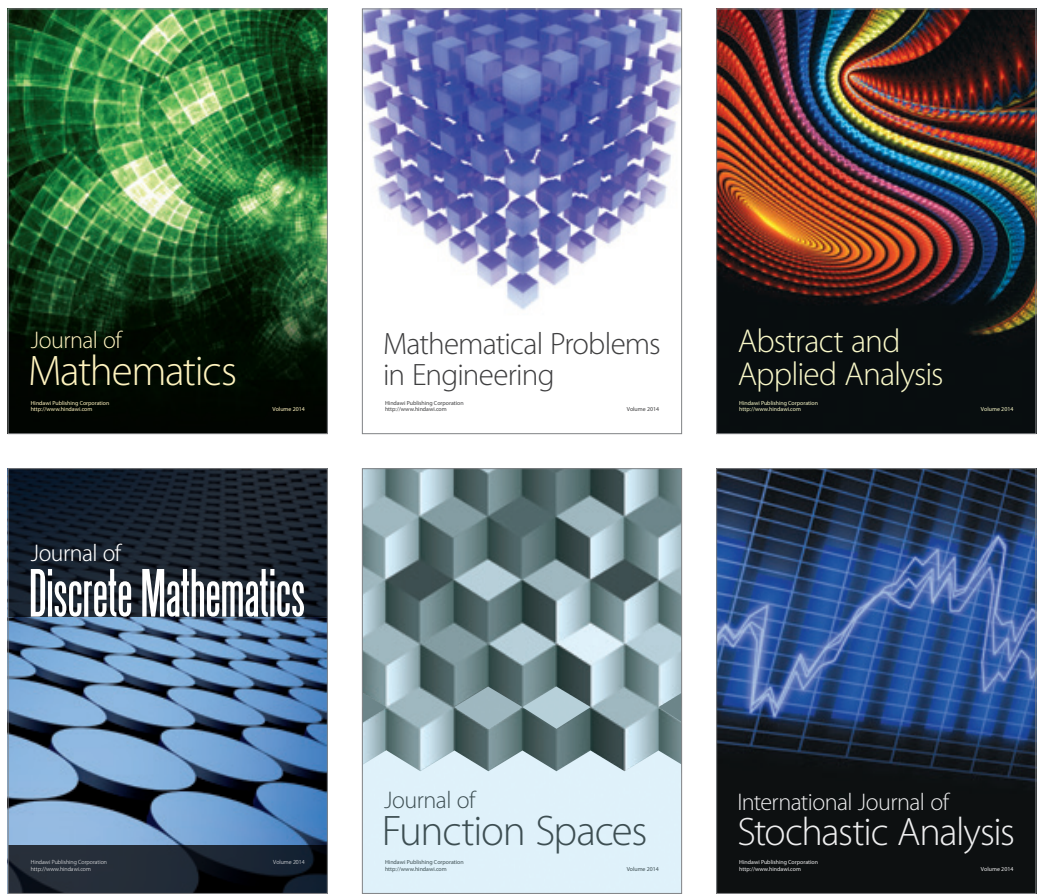

Journal of

Function Spaces

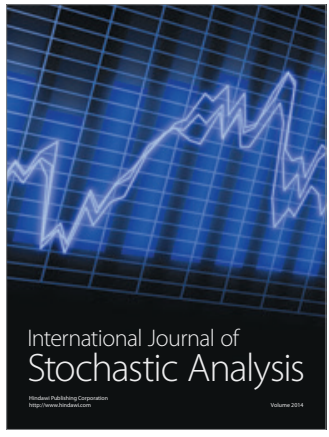

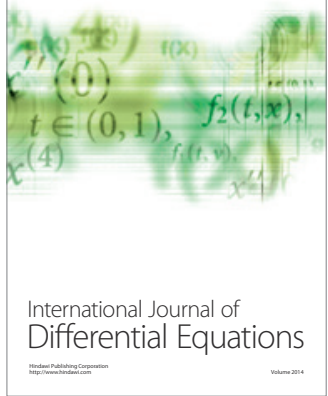
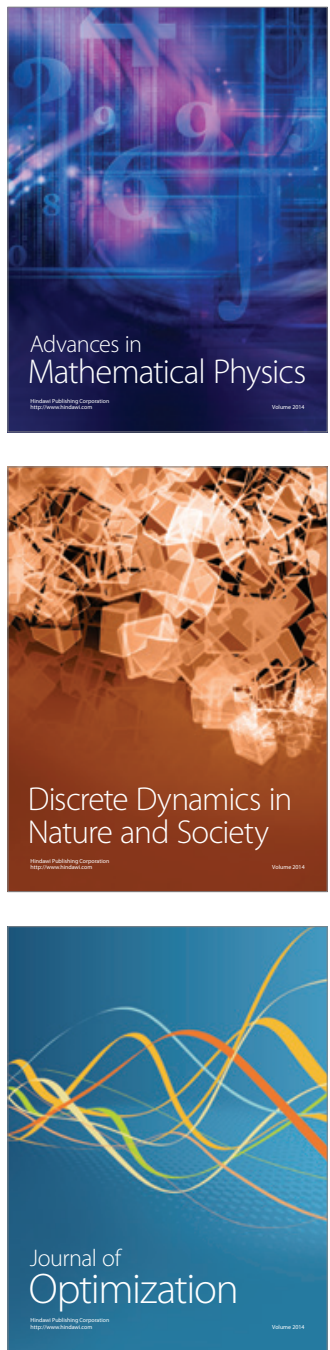